\title{
A Global Convergence of LS-CD Hybrid Conjugate Gradient Method
}

\author{
Xiangfei Yang, Zhijun Luo, and Xiaoyu Dai \\ Department of Mathematics and Econometrics, Hunan University of Humanities, Science, and Technology, Loudi 417000, China
}

Correspondence should be addressed to Zhijun Luo; ldlzj123@163.com

Received 25 June 2013; Revised 3 September 2013; Accepted 4 September 2013

Academic Editor: William J. Layton

Copyright (C) 2013 Xiangfei Yang et al. This is an open access article distributed under the Creative Commons Attribution License, which permits unrestricted use, distribution, and reproduction in any medium, provided the original work is properly cited.

Conjugate gradient method is one of the most effective algorithms for solving unconstrained optimization problem. In this paper, a modified conjugate gradient method is presented and analyzed which is a hybridization of known LS and CD conjugate gradient algorithms. Under some mild conditions, the Wolfe-type line search can guarantee the global convergence of the LS-CD method. The numerical results show that the algorithm is efficient.

\section{Introduction}

Consider the following nonlinear programs:

$$
\min _{x \in R^{n}} f(x),
$$

where $R^{n}$ denotes an $n$-dimensional Euclidean space and $f(x)$ is continuously differentiable function.

As you know, conjugate gradient method is a line search method that takes the following form:

$$
x_{k+1}=x_{k}+\alpha_{k} d_{k}, \quad k=0,1,2 \ldots,
$$

where $d_{k}$ is a descent direction of $f(x)$ at $x_{k}$ and is a stepsize obtained by some one-dimensional line search. If $x_{k}$ is the current iterate, we denote $f\left(x_{k}\right) \triangleq f_{k}, \nabla f\left(x_{k}\right) \triangleq g_{k}$, and $\nabla^{2} f\left(x_{k}\right) \triangleq G_{k}$, respectively. If $G_{k}$ is available and inverse, then $d_{k}=-G_{k}^{-1} g_{k}$ leads to the Newton method and $d_{k}=$ $-g_{k}$ results in the steepest descent method [1]. The search direction $d_{k}$ is generally required to satisfy $g_{k}^{T} d_{k} \leq 0$, which guarantees that $d^{k}$ is a descent direction of $f(x)$ at $x_{k}$ [2]. In order to guarantee the global convergence, we sometimes require $d_{k}$ to satisfy a sufficient descent condition as follows:

$$
g_{k}^{T} d_{k} \leq-c\left\|g_{k}\right\|^{2}
$$

where $c>0$ is a constant and $\|\cdot\|$ is the Euclidean norm. In line search methods, the well-known conjugate gradient method has the following form:

$$
d_{k}= \begin{cases}-g_{k}, & \text { if } k=0 \\ -g_{k}+\beta_{k} d_{k-1}, & \text { if } k \geq 1\end{cases}
$$

Different conjugate gradient algorithms correspond to different choices for the parameter $\beta_{k}$, where $\beta_{k}$ can be defined by

$$
\begin{gathered}
\beta_{k}^{\mathrm{FR}}=\frac{\left\|g_{k}\right\|^{2}}{\left\|g_{k-1}\right\|^{2}}, \quad \beta_{k}^{\mathrm{PRP}}=\frac{g_{k}^{T}\left(g_{k}-g_{k-1}\right)}{\left\|g_{k-1}\right\|^{2}}, \\
\beta_{k}^{\mathrm{DY}}=\frac{\left\|g_{k}\right\|^{2}}{d_{(k-1)}^{T}\left(g_{k-1}-g_{k}\right)}, \quad \beta_{k}^{\mathrm{LS}}=-\frac{g_{k}^{T}\left(g_{k}-g_{k-1}\right)}{d_{k-1}^{T} g_{k-1}}, \\
\beta_{k}^{\mathrm{CD}}=-\frac{\left\|g_{k}\right\|^{2}}{d_{k-1}^{T} g_{k-1}}, \quad \beta_{k}^{\mathrm{HS}}=-\frac{g_{k}^{T}\left(g_{k-1}-g_{k}\right)}{d_{k-1}^{T}\left(g_{k-1}-g_{k}\right)},
\end{gathered}
$$

or by other formulae. The corresponding methods are called FR (Fletcher-Reeves) [3], PRP (Polak-Ribiére-Polyak) [4, 5], DY (Dai-Yuan) [6], CD (conjugate descent [7]), LS (LiuStorey [8]), and HS (Hestenes-Stiefel [9]) conjugate gradient method, respectively.

Although the above mentioned conjugate gradient algorithms are equivalent to each other for minimizing strong convex quadratic functions under exact line search, they 
have different performance when using them to minimize nonquadratic functions or when using inexact line searches. For general objective function, the FR, DY, and CD methods have strong convergence properties, but they may have modest practical performance due to jamming. On the other hand, the methods of PRP, LS, and HS in general may not be convergent, but they often have better computational performance.

Touati-Ahmed and Storey [10] have given the first hybrid conjugate algorithm; the method is combinations of different conjugate gradient algorithms; mainly it is being proposed to avoid the jamming phenomenon. Recently, some kinds of new hybrid conjugate gradient methods are given in [1117]. Based on the new method, we focus on hybrid conjugate gradient methods and analyze the global convergence of the methods with Wolfe-type line search.

The rest of this paper is organized as follows. The algorithm is presented in Section 2. In Section 3 the global convergence is analyzed. We give the numerical experiments in Section 4.

\section{Description of Algorithm}

Algorithm 1.

Step 0. Initialization:

given a starting point $x_{0} \in R^{n}$, choose parameters

$$
0<\varepsilon \ll 1, \quad 0<\delta<\frac{1}{2}, \quad \delta<\sigma<1, \quad d_{0}=-g_{0} .
$$

Set $k=0$.

Step 1. If $\left\|g_{k}\right\|<\varepsilon$, stop; else go to Step 2 .

Step 2. Compute step size $\alpha_{k}$, such that

$$
\begin{gathered}
f\left(x_{k}\right)-f\left(x_{k}+\alpha_{k} d_{k}\right) \geq-\delta \alpha_{k} g_{k}^{T} d_{k}, \\
\sigma g_{k}^{T} d_{k} \leq g\left(x_{k}+\alpha_{k} d_{k}\right)^{T} d_{k} \leq 0 .
\end{gathered}
$$

Step 3. Let $x_{k+1}=x_{k}+\alpha_{k} d_{k}$; if $\left\|g_{k+1}\right\|<\varepsilon$, stop; otherwise, go to Step 4.

Step 4. Compute the search direction

$$
d_{k+1}=-g_{k+1}+\beta_{k+1}^{\mathrm{LS}-\mathrm{CD}} d_{k}
$$

where $\beta_{k}^{\mathrm{LS}-\mathrm{CD}}=\max \left\{0, \min \left\{\beta_{k}^{\mathrm{LS}}, \beta_{k}^{\mathrm{CD}}\right\}\right\}$.

Step 5 . Let $k:=k+1$, and go to Step 2 .

Throughout this paper, the following basic assumptions on the objective function are assumed, which have been widely used in the literature to analyze the global convergence of the conjugate gradient methods.

(H2.1) The objective function $f(x)$ is continuously differentiable and has a lower bound on the level set $L_{0}=\{x \in$ $\left.R^{n} \mid f(x) \leq f\left(x_{0}\right)\right\}$, where $x_{0}$ is the starting point.
(H2.2) The gradient $g(x)$ of $f(x)$ is Lipschitz continuous in some neighborhood $U$ of $L_{0}$; that is, there exists a constant $L>0$, such that

$$
\|g(x)-g(y)\| \leq L\|x-y\|, \quad \forall x, y \in U .
$$

Since $\left\{f\left(x_{k}\right)\right\}$ is decreasing, it is clear that the sequence $\left\{x_{k}\right\}$ generated by Algorithm 1 is contained in $L_{0}$.

\section{Global Convergence of Algorithm}

Now we analyze the global convergence of Algorithm 1.

Lemma 2. Suppose that assumptions (H2.1) and (H2.2) hold; the sequences $\left\{g_{k}\right\}$ and $\left\{d_{k}\right\}$ are to be generated by Algorithm 1, if $g_{k} \neq 0$ for all $k \geq 0$; then

$$
g_{k}^{T} d_{k}<0
$$

Proof. If $k=0$, then $d_{0}=-g_{o}$, and we get

$$
g_{0}^{T} d_{0}=-\left\|g_{0}\right\|^{2}<0
$$

When $k \geq 1$, multiplying $g_{k}^{T}$ by

$$
d_{k}=-g_{k}+\beta_{k}^{\mathrm{LS}-\mathrm{CD}} d_{k-1},
$$

we obtain

$$
g_{k}^{T} d_{k}=-\left\|g_{k}\right\|^{2}+\beta_{k}^{\mathrm{LS}-\mathrm{CD}} g_{k}^{T} d_{k-1} .
$$

It follows from $\beta_{k}^{\mathrm{LS}-\mathrm{CD}} \geq 0$ and $g_{k}^{T} d_{k-1} \leq 0$ that

$$
g_{k}^{T} d_{k} \leq-\left\|g_{k}\right\|^{2}<0
$$

Therefore, the result is true.

Lemma 3. Suppose that assumptions (H2.1) and (H2.2) hold, and consider any iteration of the form (2), where $d_{k}$ is a descent direction and $\alpha_{k}$ satisfies the Wolfe conditions (7). Then, the Zoutendijk condition

$$
\sum_{k=0}^{\infty} \frac{\left(g_{k}^{T} d_{k}\right)^{2}}{\left\|d_{k}\right\|^{2}}<+\infty
$$

holds.

Proof. From (7), we have

$$
(\sigma-1) g_{k}^{T} d_{k} \leq\left(g_{k+1}-g_{k}\right)^{T} d^{k} .
$$

In addition, the assumption (H2.2) gives

$$
L \alpha_{k}\left\|d_{k}\right\|^{2} \geq\left(g_{k+1}-g_{k}\right)^{T} d^{k} .
$$

Combing these two relations, we have

$$
\alpha_{k} \geq \frac{(\sigma-1)\left\|g_{k}^{T} d_{k}\right\|}{L\left\|d_{k}\right\|^{2}},
$$


TABLE 1: Test results for CD algorithm.

\begin{tabular}{lccr}
\hline Prob & $x_{0}$ & $x_{k}$ & $f_{*}$ \\
\hline 1 & $(4,5,10.1)$ & $(\mathrm{NaN}, \mathrm{NaN}, \mathrm{NaN})$ & $\mathrm{NaN}$ \\
2 & $(6.5,23)$ & $(14.92256489843370,14.92256511253481)$ & -0.99999999999993 \\
3 & $(7,11)$ & $(0.00000007501213,-0.49999972373559)$ & -0.24999999999992 \\
4 & $(2.5,11.9)$ & $(5.99999999984147,15.99997571516633)$ & -0.99999999998863 \\
5 & $(7,9.8)$ & $(\mathrm{NaN}, \mathrm{NaN})$ & $\mathrm{NaN}$ \\
6 & $(-3,-1,-3,-1)$ & $(\mathrm{NaN}, \mathrm{NaN}, \mathrm{NaN}, \mathrm{NaN})$ & 5000 \\
\hline$x_{0}:$ the initial point; $x_{k}:$ the final point; $f_{*}$ : the final value of the objective function; NI: the number of times of iteration for each problem.
\end{tabular}

TABLE 2: Test results for LS algorithm.

\begin{tabular}{|c|c|c|c|c|}
\hline Prob & $x_{0}$ & $x_{k}$ & $f_{*}$ & NI \\
\hline 1 & $(4,5,10.1)$ & $(-1.74444446043301,-1.74444445793385,6.97777777040829)$ & 4.09877659651031 & 27 \\
\hline 2 & $(6.5,23)$ & $(14.92256524764775,14.92256496282052)$ & -0.999999999999992 & 26 \\
\hline 3 & $(7,11)$ & $(-0.00000003058419,-0.49999989218958)$ & -0.24999999999999 & 34 \\
\hline 4 & $(2.5,11.9)$ & $(5.99999999851030,15.99997486954042)$ & -0.99999999998783 & 323 \\
\hline 5 & $(7,9.8)$ & $(-0.43256275978261,0.10814076312008)$ & 0.78917703640310 & 19 \\
\hline 6 & $(-3,-1,-3,-1)$ & $(2.50000015783668,2.50000010044152,5.24999984443432,-3.50000007174394)$ & -79.87499999999992 & 29 \\
\hline
\end{tabular}

$x_{0}$ : the initial point; $x_{k}$ : the final point; $f_{*}$ : the final value of the objective function; NI: the number of times of iteration for each problem.

which with $f\left(x_{k}+\alpha_{k} d_{k}\right) \leq f\left(x_{k}\right)+\delta \alpha_{k} g_{k}^{T} d_{k}$ implies that

$$
f_{k}-f_{k+1} \geq \delta \frac{(1-\sigma)\left(g_{k}^{T} d^{k}\right)^{2}}{L\left\|d^{k}\right\|^{2}} .
$$

Thus,

$$
\sum_{k=0}^{\infty} f_{k}-f_{k+1} \geq \sum_{k=0}^{\infty} \delta \frac{(1-\sigma)\left(g_{k}^{T} d^{k}\right)^{2}}{L\left\|d^{k}\right\|^{2}}
$$

Noting that $f$ is bounded below, (15) holds.

Furthermore, from Lemma 2 and (3), we can easily obtain the following condition:

$$
\sum_{k=0}^{\infty} \frac{\left\|g_{k}\right\|^{4}}{\left\|d_{k}\right\|^{2}}<+\infty
$$

Theorem 4. Suppose that $x_{0}$ is a starting point for which assumptions (H2.1) and (H2.2) hold. Consider Algorithm 1; then, one has either $g_{k}=0$ for some finite $k$ or

$$
\lim _{k \rightarrow \infty} \inf \left\|g_{k}\right\|=0 \text {. }
$$

Proof. The first statement is easy to show, since the only stopping point is in Step 3. Thus, assume that the algorithm generates an infinite sequence $\left\{g_{k}\right\}$; if the statement is false, there exists a constant $\varepsilon>0$, such that

$$
\left\|g_{k}\right\| \geq \varepsilon, \quad \forall k \geq 0
$$

From (8), we have

$$
d_{k}=-g_{k}+\beta_{k}^{\mathrm{LS}-\mathrm{CD}} d_{k-1} .
$$

Squaring both sides of the above equation, we get

$$
\left\|d_{k}\right\|^{2}+2 g_{k}^{T} d_{k}+\left\|g_{k}\right\|^{2}=\left(\beta_{k}^{\mathrm{LS}-\mathrm{CD}}\right)^{2}\left\|d_{k-1}\right\|^{2},
$$

that is,

$$
\left\|d_{k}\right\|^{2}=\left(\beta_{k}^{\mathrm{LS}-\mathrm{CD}}\right)^{2}\left\|d_{k-1}\right\|^{2}-2 g_{k}^{T} d_{k}-\left\|g_{k}\right\|^{2} .
$$

From the definitions of $\beta_{k}^{\mathrm{LS}}$, and $\beta_{k}^{\mathrm{CD}}$, we have

$$
0 \leq \beta_{k}^{\mathrm{LS}-\mathrm{CD}} \leq \beta_{k}^{\mathrm{CD}} \text {. }
$$

Thus, we can get

$$
\left\|d_{k}\right\|^{2} \leq\left(\beta_{k}^{\mathrm{CD}}\right)^{2}\left\|d_{k-1}\right\|^{2}-2 g_{k}^{T} d_{k}-\left\|g_{k}\right\|^{2}
$$

On the other hand, multiplying $g_{k}^{T}$ by

$$
d_{k}=-g_{k}+\beta_{k}^{\mathrm{LS}-\mathrm{CD}} d_{k-1}
$$

we obtain

$$
g_{k}^{T} d_{k}=-\left\|g_{k}\right\|^{2}+\beta_{k}^{\mathrm{LS}-\mathrm{CD}} g_{k}^{T} d_{k-1}
$$

Considering that $\beta_{k}^{\mathrm{LS}-\mathrm{CD}} \geq 0$ and $g_{k}^{T} d_{k-1} \leq 0$, we have

$$
g_{k}^{T} d_{k} \leq-\left\|g_{k}\right\|^{2}<0
$$

which indicates that

$$
\left(g_{k}^{T} d_{k}\right)^{2} \geq\left\|g_{k}\right\|^{4}
$$


TABLE 3: Test results for LS-CD algorithm.

\begin{tabular}{|c|c|c|c|c|}
\hline Prob & $x_{0}$ & $x_{k}$ & $f_{*}$ & NI \\
\hline 1 & $(4,5,10.1)$ & $(-1.74444443639771,-1.74444443412601,6.97777778236907)$ & 1.92102149037102 & 25 \\
\hline 2 & $(6.5,23)$ & $(14.92256501285285,14.92256521587144)$ & -0.99999999999996 & 15 \\
\hline 3 & $(7,11)$ & $(0.00000000238593,-0.49999999997767)$ & -0.25000000000000 & 23 \\
\hline 4 & $(2.5,11.9)$ & $(5.99999999856486,15.99997486532800)$ & -0.99999999998782 & 311 \\
\hline 5 & $(7,9.8)$ & $(-0.43256260505806,0.10814059874208)$ & 0.78917703640311 & 23 \\
\hline 6 & $(-3,-1,-3,-1)$ & $(2.49999974693426,2.49999983895816,5.25000008609708,-3.49999988497012)$ & -79.87499999999987 & 16 \\
\hline
\end{tabular}

$x_{0}$ : the initial point; $x_{k}$ : the final point; $f_{*}$ : the final value of the objective function; NI: the number of times of iteration for each problem.

Dividing the above inequality $(28)$ by $\left(g_{k}^{T} d_{k}\right)^{2}$, we obtain

$$
\begin{aligned}
& \frac{\left\|d_{k}\right\|^{2}}{\left(g_{k}^{T} d_{k}\right)^{2}} \leq \frac{\left(\beta_{k}^{\mathrm{CD}}\right)^{2}\left\|d_{k-1}\right\|^{2}}{\left(g_{k}^{T} d_{k}\right)^{2}}-\frac{2 g_{k}^{T} d_{k}}{\left(g_{k}^{T} d_{k}\right)^{2}}-\frac{\left\|g_{k}\right\|^{2}}{\left(g_{k}^{T} d_{k}\right)^{2}} \\
& =\frac{\left(-\left\|g_{k}\right\|^{2} / d_{k-1}^{T} g_{k-1}\right)^{2}\left\|d_{k-1}\right\|^{2}}{\left(g_{k}^{T} d_{k}\right)^{2}} \\
& -\frac{2 g_{k}^{T} d_{k}}{\left(g_{k}^{T} d_{k}\right)^{2}}-\frac{\left\|g_{k}\right\|^{2}}{\left(g_{k}^{T} d_{k}\right)^{2}} \\
& \leq \frac{\left(-\left\|g_{k}\right\|^{2} / d_{k-1}^{T} g_{k-1}\right)^{2}\left\|d_{k-1}\right\|^{2}}{\left\|g_{k}\right\|^{4}} \\
& -\frac{2 g_{k}^{T} d_{k}}{\left\|g_{k}\right\|^{4}}-\frac{\left\|g_{k}\right\|^{2}}{\left\|g_{k}\right\|^{4}} \\
& \leq \frac{\left\|d_{k-1}\right\|^{2}}{\left(d_{k-1}^{T} g_{k-1}\right)^{2}}+\frac{2}{\left\|g_{k}\right\|^{2}}-\frac{1}{\left\|g_{k}\right\|^{2}} \\
& =\frac{\left\|d_{k-1}\right\|^{2}}{\left(d_{k-1}^{T} g_{k-1}\right)^{2}}+\frac{1}{\left\|g_{k}\right\|^{2}} \text {. }
\end{aligned}
$$

Using the above inequality recursively and noting that

$$
\left\|d_{0}\right\|^{2}=-g_{0}^{T} d_{0}=\left\|g_{0}\right\|^{2},
$$

we have

$$
\frac{\left\|d_{k}\right\|^{2}}{\left(g_{k}^{T} d_{k}\right)^{2}} \leq \sum_{l=0}^{k-1} \frac{1}{\left\|g_{l}\right\|^{2}} .
$$

Then, from (23) and (35), it holds that

$$
\frac{\left(g_{k}^{T} d_{k}\right)^{2}}{\left\|d_{k}\right\|^{2}} \geq \frac{\varepsilon^{2}}{k} .
$$

Thus, it is easy to obtain

$$
\sum_{k=0}^{\infty} \frac{\left(g_{k}^{T} d_{k}\right)^{2}}{\left\|d_{k}\right\|^{2}}=+\infty
$$

This contradicts the Zoutendijk condition (15). Therefore, the conclusion holds.
TABLE 4: The performance of the LS method, CD method, and LSCD method.

\begin{tabular}{lcccc}
\hline Prob & Dim & $\begin{array}{c}\text { LS } \\
\text { NI/NF/NG }\end{array}$ & $\begin{array}{c}\text { CD } \\
\text { NI/NF/NG }\end{array}$ & $\begin{array}{c}\text { LS-CD } \\
\text { NI/NF/NG }\end{array}$ \\
\hline \multirow{3}{*}{ PEN 1 } & 100 & $51 / 142 / 92$ & $62 / 223 / 182$ & $51 / 168 / 125$ \\
& 1000 & $33 / 125 / 83$ & $52 / 181 / 165$ & $33 / 164 / 117$ \\
& 10000 & $21 / 118 / 72$ & $31 / 157 / 121$ & $21 / 132 / 102$ \\
TRIG & 100 & $305 / 399 / 398$ & $\mathrm{NaN}$ & $305 / 399 / 398$ \\
& 500 & $343 / 424 / 423$ & $\mathrm{NaN}$ & $343 / 424 / 423$ \\
ROSEX & 500 & $52 / 112 / 107$ & $92 / 267 / 238$ & $50 / 186 / 157$ \\
& 1000 & $70 / 149 / 145$ & $98 / 287 / 255$ & $70 / 246 / 183$ \\
\hline
\end{tabular}

Prob: the test problem name from [18]; Dim: the problem dimension; NI: the iterations number; NF: the function evaluations number; NG: the gradient evaluations number.

\section{Numerical Experiments}

In this section, we give the numerical results of Algorithm 1 to show that the method is efficient for unconstrained optimization problems. We set the parameters $\delta=0.3$, and $\delta=0.7$ and use MATLAB 7.0 to test the chosen problems on a PC with 2.10 GHz CPU processor, 1.0 GB RAM memory, and Linux operation system. We also use the condition $\left\|g_{k}\right\| \leq$ $10^{-6}$ or It-max $>5000$ as the stopping criterion (It-max denotes the maximal number of iterations). When the limit of 5000 function evaluations was exceeded, the run was stopped, which is indicated by "NaN." The problems that we tested are from $[17,19]$.

$$
\begin{aligned}
& \text { Prob } 1 f(x)=\left(x_{1}+3 x_{2}+x_{3}\right)^{2}+4\left(x_{1}-x_{2}\right)^{2}, \\
& \text { Prob } 2 f(x)=\sin \left(x_{1}+x_{2}\right)+\left(x_{1}-x_{2}\right)^{2}, \\
& \text { Prob } 3 f(x)=\ln \left(1+x_{1}^{2}\right)+x_{2}^{2}+x_{2}, \\
& \text { Prob } 4 f(x)=\sin \left(\pi x_{1} / 12\right) \cos \left(\pi x_{2} / 16\right), \\
& \text { Prob } 5 f(x)=e^{x_{1}}+x_{1}^{2}+2 x_{1} x_{2}+4 x_{2}^{2}, \\
& \text { Prob } 6 f(x)=x_{1}^{2}+x_{2}^{2}+2 x_{3}^{2}+x_{4}^{2}-5\left(x_{1}+x_{2}\right)-21 x_{3}+7 x_{4} .
\end{aligned}
$$

Tables 1, 2, and 3 show the computation results.

Because conjugate gradient algorithms are devised for solving large-scale unconstrained optimization problems, we chose some large-scale problems from [18] and compared the performance of the hybrid LS-CD method (Algorithm 1 in Section 2) with the LS method and CD method.

From Tables 1, 2, 3, and 4, we see that the performance of Algorithm 1 is better than that of the CD and the LS methods 
for some problems. Therefore, our numerical experiments show that the algorithm is efficient.

\section{Acknowledgments}

The authors would like to thank the anonymous referee for the careful reading and helpful comments and suggestions that led to an improved version of this paper. This work was supported in part by the Foundation of Hunan Provincial Education Department under Grant (nos. 12A077 and 13C453) and the Educational Reform Research Fund of Hunan University of Humanities, Science, and Technology (no. RKJGY1320).

\section{References}

[1] J. Nocedal and J. S. Wright, Numerical Optimization, Springer, New York, NY, USA, 1999.

[2] Y. Yuan, Numerical Methods for Nonlinear Programming, Shanghai Scientific \& Technical Publishers, Shanghai, China, 1993.

[3] R. Fletcher and C. Reeves, "Function minimization by conjugate gradients," The Computer Journal, vol. 7, pp. 149-154, 1964.

[4] E. Polak and G. Ribiére, "Note sur la convergence de méthodes de directions conjuguées," Revue Française de Recherche Opérationnelle, no. 16, pp. 35-43, 1969.

[5] B. T. Polyak, "The conjugate gradient method in extreme problems," Computational Mathematics and Mathematical Physics, vol. 9, pp. 94-112, 1969.

[6] Y. H. Dai and Y. Yuan, "A nonlinear conjugate gradient method with a strong global convergence property," SIAM Journal on Optimization, vol. 10, no. 1, pp. 177-182, 1999.

[7] R. Fletcher, "Unconstrained Optimization," in Practical Methods of Optimization, vol. 1, part 1, John Wiley \& Sons, New York, NY, USA, 2nd edition, 1987.

[8] Y. Liu and C. Storey, "Efficient generalized conjugate gradient algorithms. I. Theory," Journal of Optimization Theory and Applications, vol. 69, no. 1, pp. 129-137, 1991.

[9] M. R. Hestenes and E. Stiefel, "Method of conjugate gradient for solving linear systems," Journal of Research of the National Bureau of Standards, vol. 49, pp. 409-436, 1952.

[10] D. Touati-Ahmed and C. Storey, "Efficient hybrid conjugate gradient techniques," Journal of Optimization Theory and Applications, vol. 64, no. 2, pp. 379-397, 1990.

[11] Y. H. Dai and Y. Yuan, "An efficient hybrid conjugate gradient method for unconstrained optimization," Annals of Operations Research, vol. 103, pp. 33-47, 2001.

[12] N. Andrei, "A scaled BFGS preconditioned conjugate gradient algorithm for unconstrained optimization," Applied Mathematics Letters, vol. 20, no. 6, pp. 645-650, 2007.

[13] N. Andrei, "A hybrid conjugate gradient algorithm for unconstrained optimization as a convex combination of HestenesStiefel and Dai-Yuan," Studies in Informatics and Control, vol. 17, no. 4, pp. 55-70, 2008.

[14] Y.-H. Dai and C.-X. Kou, "A nonlinear conjugate gradient algorithm with an optimal property and an improved Wolfe line search," SIAM Journal on Optimization, vol. 23, no. 1, pp. 296320, 2013.

[15] S. Babaie-Kafaki and N. Mahdavi-Amiri, "Two modified hybrid conjugate gradient methods based on a hybrid secant equation,"
Mathematical Modelling and Analysis, vol. 18, no. 1, pp. 32-52, 2013.

[16] W. Jia, J. H. Zong, and X. D. Wang, "An improved mixed conjugate gradient method," Systems Engineering Procedia, vol. 4, pp. 219-225, 2012.

[17] M. Sun and J. Liu, "A new conjugate method and its global convergence," Journal of Information and Computing Science, vol. 8, no. 1, pp. 75-80, 2013.

[18] J. J. Moré, B. S. Garbow, and K. E. Hillstrom, "Testing unconstrained optimization software," ACM Transactions on Mathematical Software, vol. 7, no. 1, pp. 17-41, 1981.

[19] W. Hock and K. Schittkowski, "Test examples for nonlinear programming codes," Journal of Optimization Theory and Applications, vol. 30, no. 1, pp. 127-129, 1981. 


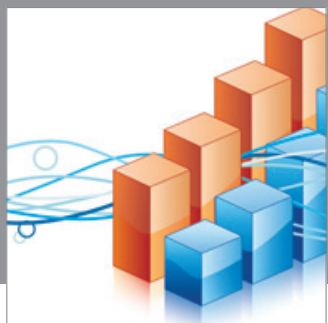

Advances in

Operations Research

mansans

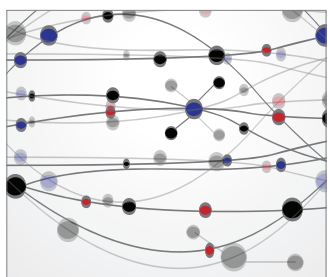

The Scientific World Journal
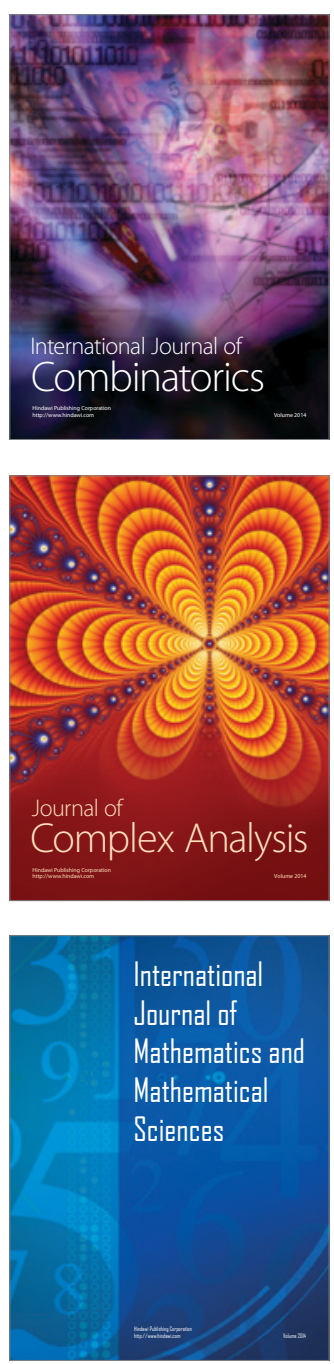
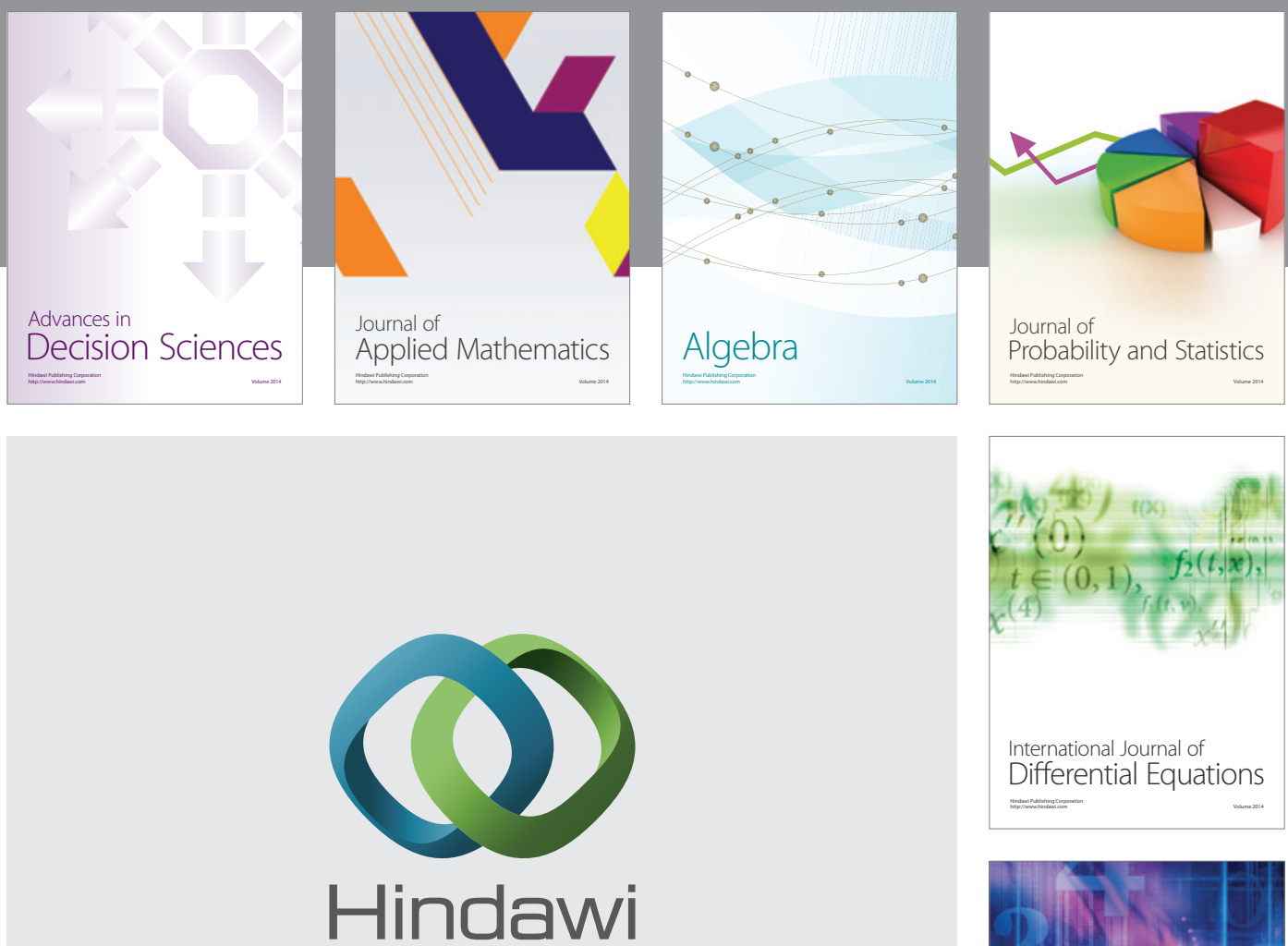

Submit your manuscripts at http://www.hindawi.com
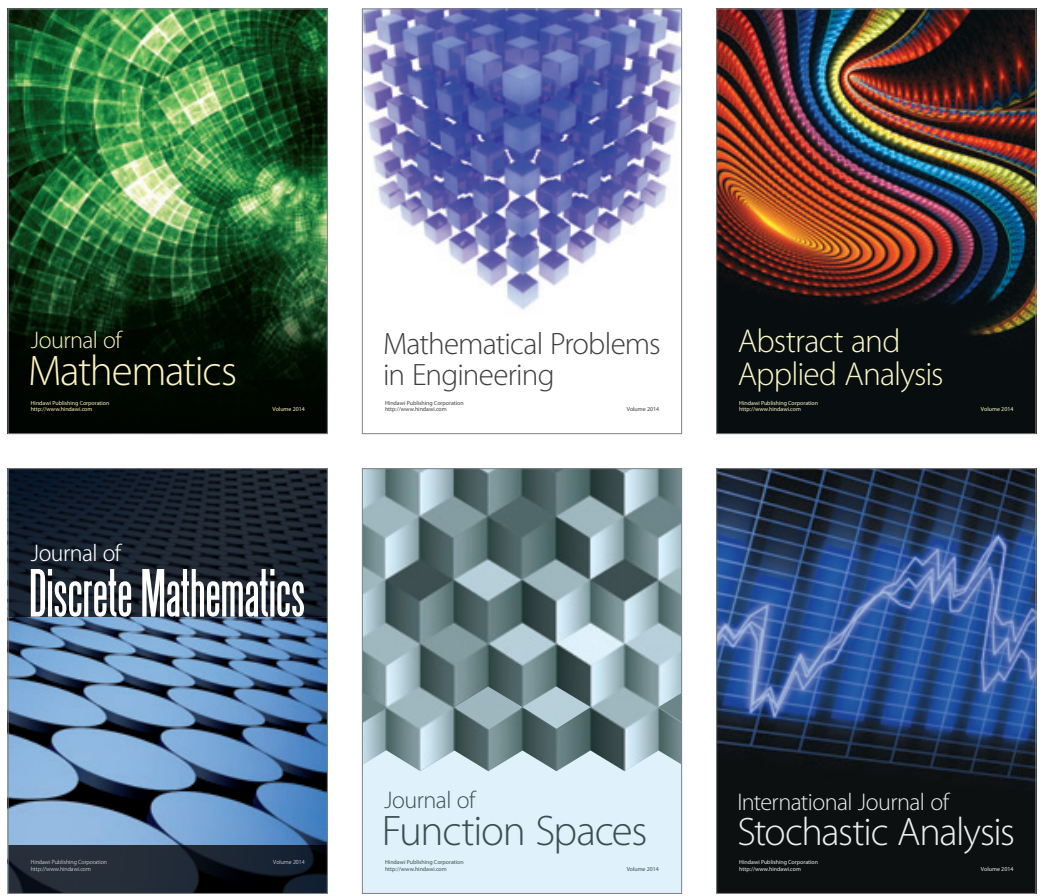

Journal of

Function Spaces

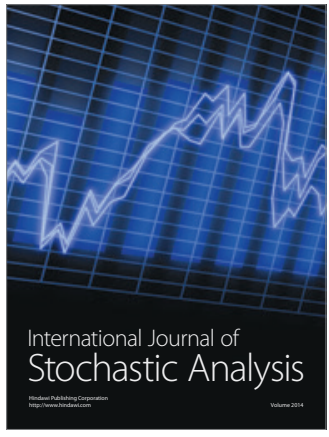

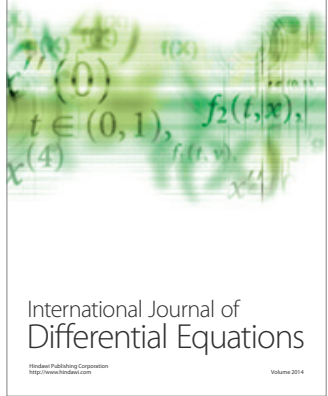
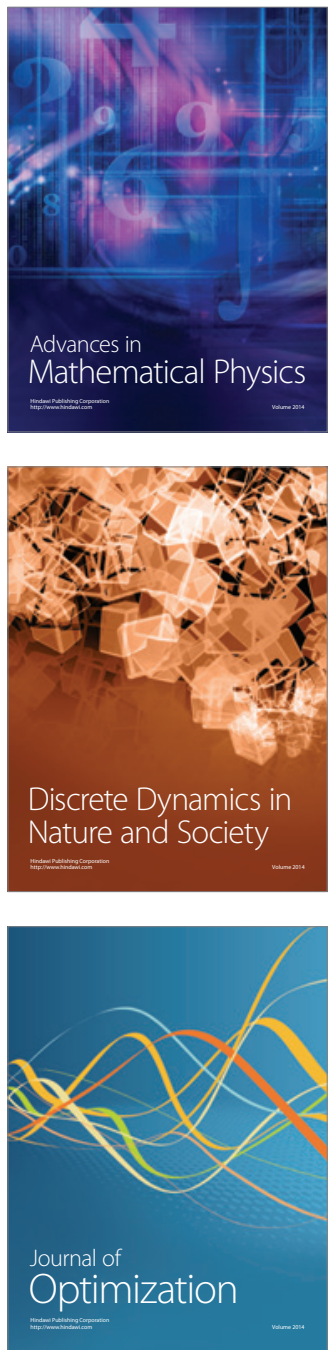